\title{
Working with adult survivors of child sexual abuse
}

\author{
Much can be learnt from what goes wrong
}

Many people who have been sexually abused as children present for help in adult life still suffering the emotional legacy of their traumatic experience.' Increasingly, some present themselves directly as victims of sexual abuse in childhood; others turn up with various psychiatric or gynaecological symptoms or general poor health-the history of sexual abuse comes to light only as treatment continues.

In these circumstances, intense feelings may arise between the doctor and patient, which offer insights that can be used to help the patient. An analysis of the doctor-patient relationship gives a glimpse of the patient's childhood relationships and how pathological these may have been. An understanding of these may improve the treatment's chance of success, helping the doctor to avoid the pitfalls that can so easily sabotage treatment.

Given the highly exploitative and damaging relationships that these patients have experienced, they find it difficult to trust their doctor. ${ }^{2}$ Thus they may respond defensively to anything even slightly reminiscent of abuse-such as an intrusive question or a well intentioned attempt to coerce them into treatment. Patients may seem oversensitive to any suggestion that what they say is not being believed or taken seriously enough. They may feel desperately let down by any perceived neglect such as being kept waiting or having a small detail forgotten. Sometimes they respond with anger; more commonly they simply fail to return for treatment.

Particularly important to this group of patients is the gender of the doctor. With male doctors they will mistrust any hint of an erotic dimension to the relationship. They also have a tendency to abdicate power to men and then feel frightened. They are likely to perceive women doctors as not "good enough" and to respond to them with anger, frustration, and disappointment. ${ }^{3}$ Their doctors' care and concern in their problems invokes grief at the lack of adequate nurturing that they received in childhood. Doctors therefore find themselves steering a fine line between being "intrusive" on the one hand and "neglectful" on the other.

Many patients grew up in families where normal social roles were grossly distorted; not only do they feel confused about their own role but they also perceive other people as confused in their roles. Some patients were abused before they had developed a consistent sense of themselves as separate people and before they had acquired a vocabulary to describe what was happening. They are particularly prone to being swamped by emotions and physical sensations and experience difficulty putting their problems into words. ${ }^{4}$
In the clinical setting these patients may seem uncertain, diffident, inappropriately friendly, flirtatious, or suspicious. They may communicate great distress but be unable to describe their symptoms, leaving the doctor wondering why they have come. Reacting to these confusions by exerting more control in the relationship is problematic as it can evoke the profound powerlessness that all childhood victims of sexual abuse experience. Doctors should, however, try to make it clear to the patient what is expected of him or her and take time to explain what they are doing.

At the time that the patients were being abused they felt trapped. Often attempts to tell the secret were disbelieved or denied or resulted in violence or the break up of their family. In their desperate search for love as adults they find themselves yet again in abusive relationships and continue to relate to the world and their present problems with a despairing acceptance. It is tempting for the concerned infuriated professional to respond with action, but doctors should remain alert to the likelihood that they too may be perceived by the patient as pushing them into doing something against their will. There is a need to respond sensitively and flexibly to facilitate these patients' development from victims to survivors. ${ }^{6}$

For survivors of sexual abuse, managing their rage and hatred is a crucial task. Many of them express strong feelings of hatred towards the offender or parent who didn't notice or direct it more generally against all men or "society," which failed to protect them. It may be displaced on to the patient's partner or children and may be prominent in their attitude towards professionals. Their hate may also be massively projected-that is, the patient feels hated by everyone and consequently lives a restricted life. ${ }^{4}$ Survivors who turn to doctors for help are often those who are unable to recognise or express their anger and hatred. These feelings are associated with guilt and low self esteem, and the risk of self harm is high. One of the doctors' problems is to recognise and contain the anger when it is directed towards them without retaliating. ${ }^{7}$

The sexual molestation of a child or adolescent violates normal physical and psychological development. Sex becomes linked with violence, fear, and guilt, and some experience the depths of shame because they were sexually excited by the abuse - particularly if this was exploited by the perpetrator. ${ }^{8}$ Others find it hard to acknowledge that they have positive as well as negative feelings towards the perpetrator and may on one level have enjoyed the sexual relation- 
ship and the "specialness" of the attention that they received. Many patients cope by avoiding or denying their sexual feelings. ${ }^{9}$

Others, feeling uncomfortable and unsafe with their sexuality, use it in chaotic, inappropriate, and self defeating ways. In working through feelings of shock, revulsion, and anger and re-examining issues of power, violence, and sexism within society doctors may find their own sexual relationships affected and may go through a stage where they see abuse everywhere. ${ }^{10}$ They may find themselves "turned on" as much as "turned off" by accounts of the abuse and mistrust their own motives.

The power and intensity of emotion experienced by both patient and doctor in the therapeutic relationship can be disturbing. Some of these feelings may remain unconscious but get acted on to the detriment of treatment-for example, doctors may inappropriately discharge or over investigate the patient. If the doctor can try to understand what is going on in his or her relationship with the patient and recognise the feelings that emerge as important clues about the patient's childhood then managing the patient will be far more rewarding and the patient's prognosis will be substantially improved.

I thank Dr Chris Whyte for his many helpful comments.

Senior Registrar in Psychotherapy,

PENELOPE CAMPLING

Humberstone Grange Clinic,

Leicester LE5 0TA

1 Sheldon $\mathrm{H}$. Child sexual abuse in adult female psychotherapy referrals: incidence and implicatio for treatment. Br f Psychiatry 1988;152:107-11.

Hall Z. Understanding women in distress. London: Tavistock/Routledge, 1989

3 Gardener F. Psychotherapy with adult survivors of child sexual abuse. British fournal Psychotherapy 1990;6:285-94

4 MacCarthy B. Are incest victims hated? Psychoanalytic Psychotherapy 1988;3:113-20.

5 Fountain DM, Moore B. Therapy for adult victims of childhood sexual abuse in a district setting Psychiatric Bulletin 1989;13:437-9.

6 Davenport S, Sheldon H. From victim to survivor. Changes 1987;5:379-82.

7 Winnicott DW. Hate in the countertransference. Int $\mathcal{F}$ Psychoanal 1949;30:60-7

Cahill C, Llewelyn SP, Pearson C. Treatment of sexual abuse which occurs in childhood: A reviewo Brf Clin Psychol 1991;30:1-12.

9 John D. Beyond sexual abuse: therapy with women who were victims in childhood. Chichester: Wiley, 1980

10 Perry J. Sexual abuse-the counsellor's process. Changes. 1989;7(3):93-4.

\section{How general practitioners can help subfertile couples}

\section{Support, early investigation, and referral}

Subfertility affects one couple in $10^{1}$ and may cause as deep and enduring a sense of loss as many chronic illnesses. ${ }^{2}$ Commissioning authorities and general practitioners should therefore take subfertility seriously. Fortunately, the condition is starting to receive the attention that it deserves, and two important documents have recently been published on its management. ${ }^{34} \mathrm{~A}$ database of clinical trials incorporating structured overviews and meta-analyses has been established jointly in Leeds and Hamilton, Ontario, and is funded by the Yorkshire research and development programme and the Canadian Royal Commission on Reproductive Technologies.

Where do general practitioners fit into the diagnosis and management of infertility? Firstly, general practitioners can provide the emotional support that most infertile couples need before, during, and after treatment, whether successful or not. Subfertility evokes powerful and complex emotions, which can make it difficult for couples to assimilate spoken information. Yet such information is crucial if they are to make fully informed decisions about their treatment. Although hospital clinics may provide written information and dedicated counsellors to help patients over particularly difficult times (for example, when assisted conception has failed), this cannot replace the general practitioner, with whom the couple are likely to have a longstanding relationship.

It is the general practitioner who will guide the couple to secondary care, having already listened to their history, examined them, and undertaken initial investigations. He or she can smooth the progress of investigation and act as a valuable source of advice and explanation during what may be complex and intensive treatments, ensuring that crucial information, such as the risks and consequences of twins and higher order births, have been fully assimilated.

Finally, it may be the general practitioner who has to help the couple face and come to accept their permanent childlessness. Such involvement was much hindered by the requirements of confidentiality in the Human Embryology and Fertilization Act, which, before the recent amendment, kept general practitioners unaware of their patients' treatment. General practitioners can help by reducing the number of journeys to distant clinics, as well as acting as the patient' advocate and providing continuity.

The main issues for general practitioners concern how much investigation they should carry out, when and to whomes they should refer the patient (local hospital or tertiary centre)? and how much they should be involved in on going treatmento (for example, giving drugs to stimulate ovulation). Far toos many patients are brought back time and again to outpatiento clinics where they are subjected to an unending series of investigations, ${ }^{5}$ most of which have little hope of improving their chance of success. General practitioners, in their role aso purchaser and patient's advocate, are responsible for guard-ing against this. Genuine remediable causes are found in lesso. than half the cases of subfertility. ${ }^{6}$ The general practitioner's role is therefore twofold: firstly, to enable the couple to find out whether a specific cause exists and to refer accordinglyo and, secondly, to give patients the information that they need to decide when, if ever, they wish to pursue assistedo conception procedures.

Known causes of subfertility can be divided into failure to요 ovulate, defective sperm, and tubal damage. General practi-N tioners can exclude failure to ovulate by taking a carefully timed blood sample for measurement of the progesterone concentration. This must be interpreted with reference to the date of the succeeding menstrual period. Semen analyses can $N_{\omega}$ exclude most abnormalities of the sperm; if the sperm count iso initially low it should be repeated. Ideally, the sperm shouldo be analysed by the laboratory connected to the centre to which the patients will be referred if further investigation or treatment is necessary. Tubal damage can be assessed laparoscopically, and a set protocol and method of documenting the findings should be used. Couples prefer to be seen in designated subfertility clinics rather than general outpatientoclinics.

Some regions have drawn up protocols for investigation and treatment by general practitioner, nonspecialist, and specialist hospitals. A randomised study in Grampian has shown that published guidelines are effective in improving referral practice and cutting down unnecessary investigations (C Emslie et al, unpublished data). 